\title{
Iodine Deficiency Hypothyroidism
}

National Cancer Institute

\section{Source}

National Cancer Institute. Iodine Deficiency Hypothyroidism. NCI Thesaurus. Code C132053.

Hypothyroidism due to insufficient intake of iodine. 